\title{
Laparoscopic repair of vaginal vault prolapse by lateral suspension with mesh
}

\author{
Jean Dubuisson · Isabelle Eperon · Patrick Dällenbach • \\ Jean-Bernard Dubuisson
}

Received: 14 June 2012/ Accepted: 11 September 2012/Published online: 22 September 2012

(C) Springer-Verlag 2012

\begin{abstract}
Background To evaluate the long-term outcomes of laparoscopic lateral suspension using mesh reinforcement for symptomatic posthysterectomy vaginal vault prolapse.

Materials and methods We analyzed in a prospective cohort study all the women treated by laparoscopic lateral suspension with mesh for symptomatic vaginal vault prolapse between January 2004 and September 2010. In this procedure, the mesh is laterally suspended to the abdominal wall, posterior to the anterior superior iliac spine. We performed systematic follow-up examinations at 4 weeks, 6 months and yearly postoperatively. Clinical evaluation of pelvic organ support was assessed by the pelvic organ prolapse quantification (POP-Q) grading system. Main outcome measures were recurrence rate, reoperation rate for symptomatic recurrence or de novo prolapse, mesh erosion rate, reoperation rate for mesh erosion, total reoperation rate.

Observations and results of the 73 patients seen at a mean 17.5 months follow-up, recurrent vaginal vault prolapse was registered in only one woman (success rate of $98.6 \%$ ). When considering all vaginal sites, we observed a total of 13 patients with recurrent or de novo prolapse $(17.8 \%)$. The non-previously treated posterior compartment was involved in eight cases (new appearance rate of $11 \%$ ). Of these 13 women, only 6 were symptomatic, requiring surgical management (reoperation rate for genital
\end{abstract}

J. Dubuisson $(\varangle)$ · I. Eperon · P. Dällenbach

Perineology Unit, Division of Gynecology, Department of

Obstetrics and Gynecology, University Hospitals of Geneva,

30 Boulevard de la Cluse, 1211 Geneva, Switzerland

e-mail: neajdubuisson@yahoo.fr

J.-B. Dubuisson

University of Geneva, Geneva, Switzerland prolapse of $8.2 \%$ ). Four patients presented with mesh erosion into the vagina $(5.5 \%)$. Two required partial vaginal excision of the mesh in the operating room $(2.7 \%)$. There were no mesh-related infections. The total reoperation rate was $11 \%$.

Conclusion Laparoscopic lateral suspension with mesh interposition is a safe and effective technique for the treatment of vaginal vault prolapse. This approach represents an alternative procedure to the laparoscopic sacrocolpopexy.

Keywords Vaginal vault prolapse - Laparoscopy · Lateral colposuspension $\cdot$ Polypropylene mesh

\section{Introduction}

Genital prolapse is a common condition and may affect about half of parous women. Ten to $20 \%$ of these women are symptomatic [1]. Pelvic floor disorders occur in about $38 \%$ of women who previously had hysterectomy [2]. The overall incidence of vaginal vault prolapse after hysterectomy is estimated to range from 0.2 to $1 \%$ [3-5]. The cumulative incidence of vaginal vault repair with a mean follow-up period of 13 years was $0.5 \%$ in our institution [3]. A surgical approach is proposed in case of poor quality of life and failure of physiotherapy exercises. Goals of surgical treatment are to improve symptoms and repair the pelvic support anatomy.

Many approaches have been described in the literature during the past decade. The development of laparoscopic surgery techniques [6] and more recently the advances in robotic surgery [7] have minimized the morbidity associated with transabdominal procedures. Laparoscopy allows optimal access to the pelvic floor with a perfect view. 
Sacrocolpopexy is the most published and used of the laparoscopic procedures $[8,9]$. In this technique, the mesh is fixed to the anterior longitudinal ligament at the promontory. However, this technique is associated with rare but serious morbidity, with case reports of vascular injuries and nervous damage to sacral nerve roots resulting in chronic constipation and pain $[10,11]$.

The technique of laparoscopic lateral suspension with mesh was first reported by Dubuisson [12] in 1998 for the treatment of genital prolapse. The laterosuspension avoids both the risk of vascular injury and nerve damage of laparoscopic sacrocolpopexy. Several modifications were described but the operation is now following a standard surgical procedure, which was not modified since 2003. The latest results were recently published [13, 14]. The technique is different from the vesicovaginal suspension described by Kapandji [15]. The Kapandji procedure was abandoned since decades because of high recurrences of postoperative enterocele. In this article, we restricted the analysis to women having a laparoscopic lateral suspension for posthysterectomy vaginal vault prolapse. The objective was to evaluate the safety and the efficiency of the surgical procedure in these cases.

\section{Materials and methods}

\section{Patients}

Between January 2004 and September 2010, we prospectively analyzed all patients with symptomatic posthysterectomy vaginal vault prolapse who were treated by laparoscopic lateral suspension with mesh at the Geneva University Hospitals. All patients underwent a standardized colposuspension technique by the same surgeon (JBD) after informed consent had been obtained. The study was not submitted to the ethical committee because the follow-up described below was our usual practice. The following data were collected for each patient: age, body mass index (BMI), vaginal parity, history of previous surgery for genital prolapse or urinary incontinence and type of hysterectomy performed. All women had a standardized preoperative prolapse assessment using the POP-Q System [16]. Pelvic disorders, sexual dysfunctions, functional urinary and digestive symptoms were detailed at each visit using questionnaires. These questionnaires were not validated questionnaires, simply an assessment of presence or absence of these symptoms. Urodynamic investigation was performed only in patients with stress urinary incontinence or urinary retention problems. Additional surgical procedures, operating time, perioperative complications and duration of hospitalization were collected for each patient. It was a delayed complication if it occurred more than
4 weeks after surgery. In case of complications related to the use of prosthetic meshes, we followed the French Guidelines for clinical practice [17]. Mesh erosions were managed both conservatively and surgically. Asymptomatic patients (small exposed area without obvious inflammatory response) were treated with transvaginal estrogen. Symptomatic or large erosions required surgical excision by vaginal route. All operations were performed under antibiotic coverage, according to the standard of our institution. Patients had standardized follow-up examinations by the medical team at 4 weeks, 6 months and yearly post-surgery. Pelvic examination included the POP-Q classification. For each patient, examination findings at the last visit were used for the long-term follow-up data. Recurrent prolapse was defined as postoperative stage $\geq 2$, whatever the symptoms described. It was a late recurrence if it occurred more than 12 months after surgery.

\section{Surgical technique}

The procedure was performed under general anesthesia. The surgical technique was divided into two principle steps. The first step was the dissection and the mobilization of the bladder and the rectum from the apex of the vagina. The second step was the application of the surgical mesh with the principle of tension-free suspension.

Step 1 It started with a complete adhesiolysis in case of posthysterectomy intestinal adhesions with the vaginal vault scar. The vesicovaginal space was found between the bladder and the anterior vaginal wall in the fascia plane. If needed, the bladder was filled in a retrograde manner with 200-300 $\mathrm{ml}$ of a blue-colored serum to facilitate the dissection. Posteriorly, the rectovaginal septum was opened distally to the perineal body and the anorectal junction.

Step 2 A polypropylene mesh (Gynemesh $25 \times 25 \mathrm{~cm}$, Ethicon, Inc., Somerville, NJ) was cut to obtain two long arms (15-20 mm wide) and a rectangular piece $(4-7 \mathrm{~cm}$ wide). The mesh was fashioned over the dissected anterior and posterior walls of the vagina and fixed with separated number 0 polyester sutures. A 5-mm skin incision was made on both sides at $2 \mathrm{~cm}$ above the iliac crest and $4 \mathrm{~cm}$ posterior to the anterior superior iliac spine. By a retroperitoneal way, a laparoscopic grasping instrument was introduced through the incision and oriented under the round ligament till the corresponding free arm (Fig. 1). The mesh was grasped and laterally pulled out slowly by the same way until having a satisfactory tension. The mesh was suspended without suture according to the "tension-free" repair principle. A satisfactory suspension was obtained if the mesh was "horizontal" at laparoscopy. The mesh was then cut at the level of the skin. Then, the peritoneum was closed over the mesh to completely retroperitonealize the graft. 


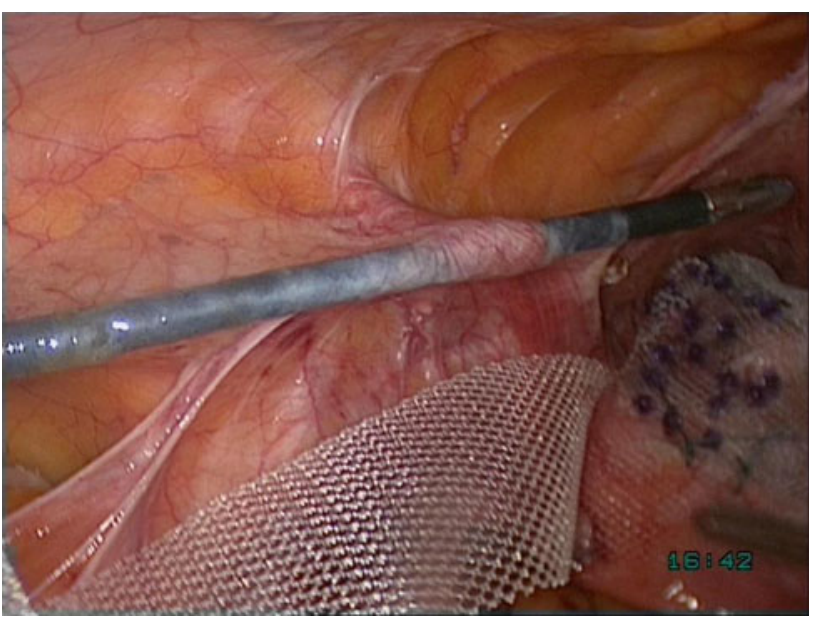

Fig. 1 Retroperitoneal passage with the forceps before the lateral placement of the mesh

\section{Observations and results}

During the study period, a total of 73 patients had laparoscopic lateral suspension with mesh for the treatment of symptomatic vaginal vault prolapse. The mean age of patients was 63 years (range 39-83), mean parity was 2 (range 0-5) and mean BMI was 26.7 (range 18-38). Prior hysterectomy was performed by laparotomy in 36 cases $(49.3 \%)$, by vaginal route in 31 cases $(42.5 \%)$, by laparoscopy in 3 cases $(4.1 \%)$, by vaginal laparoscopically assisted access in 2 cases (2.7 \%) (unknown in 1 case). Thirty-one patients had previous surgery for genital prolapse $(42.5 \%)$. Of these patients, six had two operations and two had more than two operations. Sixteen patients had previous surgery for urinary incontinence $(21.9 \%)$. Of these patients, one had two operations. The mean length of postoperative follow-up was 17.5 months (range 1-67). Pre- and postoperative symptoms are described in Table 1. Many of the symptoms were improved after surgery. Thirty-one patients had a regular sexual activity before surgery $(42.5 \%)$. Three patients who had preoperative dyspareunia were ameliorated after surgery. One patient developed de novo dyspareunia. No perioperative complications were associated with this case. There was no report

Table 1 Functional results

\begin{tabular}{lll}
\hline Symptoms & $\begin{array}{l}\text { Preoperative } \\
n(\%)\end{array}$ & $\begin{array}{l}\text { Review at mean } \\
17.5 \text { months, } n(\%)\end{array}$ \\
\hline Vaginal pressure & $62(84.9)$ & $4(5.5)$ \\
Overactive bladder & $19(26.0)$ & $3(4.1)$ \\
Voiding dysfunction & $12(16.4)$ & $2(2.7)$ \\
Stress urinary incontinence & $23(31.5)$ & $4(5.5)$ \\
Constipation & $22(30.1)$ & $4(5.5)$ \\
\hline
\end{tabular}

Table 2 Anatomical results

\begin{tabular}{lcc}
\hline Stage & Preoperative $n(\%)$ & $\begin{array}{l}\text { Postoperative at mean } \\
17.5 \text { months, } n(\%)\end{array}$ \\
\hline Anterior compartment (cystocele) & $62(84.9)$ \\
0 & $7(9.6)$ & $9(12.3)$ \\
I & $2(2.7)$ & $2(2.7)$ \\
II & $28(38.3)$ & $0(0)$ \\
III & $35(47.9)$ & $0(0)$ \\
IV & $1(1.4)$ & \\
Middle compartment (vault and enterocele) & $66(90.4)$ \\
0 & $0(0)$ & $3(4.1)$ \\
I & $21(28.8)$ & $3(4.1)$ \\
II & $24(32.9)$ & $1(1.4)$ \\
III & $27(37.0)$ & $0(0)$ \\
IV & $1(1.4)$ & \\
Posterior compartment (rectocele) & $58(79.5)$ \\
0 & $12(16.4)$ & $6(8.2)$ \\
I & $13(17.8)$ & $9(12.3)$ \\
II & $35(47.9)$ & $0(0)$ \\
III & $13(17.8)$ & $0(0)$ \\
IV & $0(0)$ &
\end{tabular}

of de novo incontinence. Anatomical results are detailed in Table 2.

Concomitant procedures were performed at the surgeon's discretion in 34 patients: 25 Burch colposuspensions, 2 suburethral slings, 7 posterior perineal repairs. Extensive adhesiolysis was necessary in 30 cases.

The mean time for the complete procedure was $193 \mathrm{~min}$ (range 90-300). The mean hospital stay was 4.4 days.

No laparoconversion was necessary. One bladder perforation occurred during dissection requiring peroperative suture and postoperative catheterization for 7 days. Recovery was uneventful. One patient developed a documented low urinary tract infection treated by oral antibiotics. One patient developed a deep venous thrombosis. There was no blood transfusion. Eight delayed complications were observed:

- Four vaginal mesh erosions (5.5\%), two of them $(2.7 \%)$ were treated by partial vaginal excision of the mesh in the operating room with no event at follow-up

- One asymptomatic suburethral sling erosion

- Three vaginal cuff granulomas treated by section of the exteriorised stitch in the office.

All erosions were observed more than 1 year after surgery.

There was no loss of follow-up. One patient with cirrhosis died of liver failure during the 2 years follow-up.

Vaginal apex was well supported postoperatively in 72 of the 73 patients initially presenting with symptomatic 
Table 3 Details on recurrent or new cases of vaginal prolapse

$C$ cystocele, $V$ vault prolapse, $R$ rectocele, $E$ enterocele

\begin{tabular}{lllll}
\hline Patients & Stage & Details & Symptoms & Surgical treatment \\
\hline 1 & C2 V1 R2 & Median cystocele & - & 0 \\
& & Low rectocele & & \\
2 & C0 V0 R2 & Low rectocele & + & Vaginal surgery \\
3 & C0 V1 R2 & Low rectocele & - & 0 \\
4 & C0 V0 R2 & High rectocele & + & Laparotomy \\
5 & C2 V0 R0 & Lateral cystocele & - & 0 \\
6 & C0 V0 R2 & Low rectocele & - & 0 \\
7 & C0 V0 R2 & Low rectocele & + & Vaginal surgery \\
8 & C0 V0 E2 & Enterocele & + & Laparoscopy \\
9 & C0 V1 E3 & Enterocele & + & Laparoscopy \\
10 & C0 V0 R2 & Low rectocele & - & 0 \\
11 & C0 V0 R2 & Low rectocele & - & 0 \\
12 & C0 V0 E2 & Enterocele & + & Vaginal surgery \\
13 & C0 V2 R2 & Vault & - & 0 \\
& & Low rectocele & & \\
\hline
\end{tabular}

apical prolapse (success rate of $98.6 \%$ ). When considering all vaginal sites, we observed a total of 13 women $(17.8 \%)$ with recurrent or de novo prolapse (Table 3 ). The nontreated lower part of the posterior wall or perineal insufficiency was involved in 8 of the 13 cases (new appearance rate of $11 \%)$. None of these patients had had a posterior colpoperineorrhaphy during the procedure. No recurrence occurred at 1-month follow-up. Four patients had a new prolapse at 6-months follow-up. The vaginal vault prolapse recurrence was observed at 1-year follow-up. A total of six patients $(8.2 \%)$ underwent a reoperation because of symptomatic prolapse. There was no standardized procedure to treat these patients. Recurrences were treated either by laparoscopy or by vaginal route. One of the six patients required a laparotomy because of extensive intestinal adhesions. None of the six patients experienced recurrence of their prolapse after the reoperation. The total reoperation rate was $11 \%(8 / 73)$.

\section{Discussion}

Initial reports have shown the feasibility and the effectiveness of the laparoscopic lateral colpo-uterine suspension with mesh reinforcement in the treatment of genital prolapse [12]. Relevant surgical indications are cystocele and/or hysterocele [13]. The procedure is not indicated in case of predominant rectocele and/or pelvic floor insufficiency. The suspension axis of lateral meshes may lead to enterocele or Douglas pouch hernia in case of perineal muscles insufficiency or descent of the upper part of the rectum. This could explain that the most common site of de novo prolapse in the design study was the posterior compartment. No recurrences were observed in the cases with associated colpoperineorrhaphy. Therefore, we believe that an associated posterior colpoperineorrhaphy could prevent further posterior compartment prolapses.

Vaginal vault prolapse repair remains a real challenge for urogynecologists. Tissues are weakened and cleavage planes are difficult to find. Those conditions increase surgical failures. The use of the laparoscopic lateral colposuspension can provide an alternative surgical approach with favorable cure rates when compared with other procedures. In a prospective randomized trial involving 108 patients with a 2-year follow-up, Maher [18] reported a $23 \%$ recurrence rate for laparoscopic sacrocolpopexy as compared with a $57 \%$ recurrence rate for transvaginal mesh procedure $(p<0.001)$. In a systematic review, Nygaard [11] reported a median reoperation rate for prolapse recurrence of $4.4 \%$ after abdominal sacrocolpopexy (range 0-18.2\%) during follow-up intervals that ranged from 6 months to 3 years. The success rate, when defined as lack of apical prolapse post-operatively, ranged from 78 to $100 \%$ and when defined as no postoperative prolapse, from 58 to $100 \%$ [11]. In laparoscopy, the success rate, when defined as no vaginal vault recurrence, ranged from 92 to $95 \%$ in recent reviews $[19,20]$. In a long-term review of laparoscopic sacrocolpopexy, Higgs [20] had an overall reoperation rate of $16 \%$ with a reoperation rate of $11 \%$ for symptomatic prolapse. Our observational study shows encouraging results with an $82.2 \%$ cure rate at a mean 17.5 months follow-up and a reoperation rate for symptomatic prolapse of $8.2 \%$. Anatomical restoration of the vaginal vault was successful in $98.6 \%$ of the patients. These findings confirm the good functional and anatomic outcomes obtained with laparoscopic repair using mesh [18]. 
Common complications of pelvic organ prolapse repair using mesh are vaginal mesh erosions and consecutive infections. It is more frequently observed with transvaginal placed meshes [21]. Based on evaluation of recent adverse reports, the Food and Drug Administration (FDA) provides recommendations and finally indicates: "the FDA has not seen conclusive evidence that using transvaginally placed mesh in pelvic organ prolapse repair improves clinical outcomes any more than traditional pelvic organ prolapse repair that does not use mesh, and it may expose patients to greater risk" [22]. We believe that these recommendations are not applicable to laparoscopic mesh reinforcement. In our experience, we have not encountered serious mesh infections. The rate of erosion was $5.5 \%$ in this study with no serious related infections. The overall mesh erosion rate published in our previous report was $3.4 \%$ (13 of the 377 patients) [14]. All the patients were treated by excision of the eroded mesh followed by simple closure of the vagina. These findings were consistent with the current literature. Mesh erosion rates with sacrocolpopexy range from 2 to $10 \%$ [23]. The majority of studies assessing mesh erosion have been performed on abdominal sacrocolpopexy, with heterogeneous data analysis mixing uterine preservation or concomitant hysterectomy and posthysterectomy groups. In case of minimally invasive sacrocolpopexy (conventional or robotic-assisted laparoscopy), Tan-Kim [23] reported a $5 \%$ rate of mesh erosion in the posthysterectomy group.

Our study has some limitations. It is a single institution experience with only one surgeon that may limit the generalizability of the findings and there is no control group. However, the strength of this study is that it is a prospective series of consecutive patients with a standardized follow-up assessment.

\section{Conclusions}

Laparoscopic lateral suspension with mesh is a safe and effective procedure for the treatment of vaginal vault prolapse. It represents an alternative of laparoscopic sacrocolpopexy to restore the anatomy of the vaginal apex. A posterior colpoperineorrhaphy must be associated in case of perineal insufficiency to avoid the appearance of a new prolapse.

Conflict of interest We declare that we have no conflict of interest.

\section{References}

1. Beck RP, McCormick S, Nordstrom L (1991) A 25-year experience with 519 anterior colporrhaphy procedures. Obstet Gynecol 78(6):1011-1018
2. Hendrix SL, Clark A, Nygaard I, Aragaki A, Barnabei V, McTiernan A (2002) Pelvic organ prolapse in the women's health initiative: gravity and gravidity. Am J Obstet Gynecol 186(6): 1160-1166

3. Dällenbach P, Kaelin-Gambirasio I, Jacob S, Dubuisson JB, Boulvain M (2008) Incidence rate and risk factors for vaginal vault prolapse repair after hysterectomy. Int Urogynecol J Pelvic Floor Dysfunct 19(12):1623-1629

4. Barrington JW, Edwards G (2000) Posthysterectomy vault prolapse: review. Int Urogynecol J Pelvic Floor Dysfunct 11(4): 241-245

5. Cruikshank SH, Cox DW (1990) Sacrospinous ligament fixation at the time of transvaginal hysterectomy. Am J Obstet Gynecol 162(6):1611-1615 (discussion 1615-1619)

6. Wattiez A, Mashiach R, Donoso M (2003) Laparoscopic repair of vaginal vault prolapse: review. Curr Opin Obstet Gynecol 15(4):315-319

7. Elliott DS, Krambeck AE, Chow GK (2006) Long-term results of robotic assisted laparoscopic sacrocolpopexy for the treatment of high grade vaginal vault prolapse. J Urol 176(2):655-659

8. Maher C, Feiner B, Baessler K, Adams EJ, Hagen S, Glazener CM (2010) Surgical management of pelvic organ prolapse in women: review. Cochrane Database Syst Rev 4:CD004014

9. Ganatra AM, Rozet F, Sanchez-Salas R, Barret E, GalianoM, Cathelineau X, et al. (2009) The current status of laparoscopic sacrocolpopexy: a review. Eur Urol. 55(5):1089-103. Epub 2009 Feb 4, Review

10. Possover M, Lemos N (2011) Risks, symptoms, and management of pelvic nerve damage secondary to surgery for pelvic organ prolapse: a report of 95 cases. Int Urogynecol J 22(12): $1485-1490$

11. Nygaard IE, McCreery R, Brubaker L, Connolly A, Cundiff G, Weber AM et al (2004) Pelvic floor disorders network. Abdominal sacrocolpopexy: a comprehensive review. Obstet Gynecol 104(4):805-823

12. Dubuisson JB, Chapron C (1998) Laparoscopic iliac colpo-uterine suspension for treatment of genital prolapse using two meshes. A new operative technique. J Gynecol Surg 14:153-159

13. Dubuisson JB, Yaron M, Wenger JM, Jacob S (2008) Treatment of genital prolapse by laparoscopic lateral suspension using mesh: a series of 73 patients. J Minim Invasive Gynecol 15(1):49-55

14. Eperon I, Luyet C, Yaron M, Dubuisson J, Dubuisson JB. (2011) Laparoscopic management of genital prolapse by lateral suspension using mesh: a series of 377 patients. Rev Med Suisse. 26;7(314):2084, 2086-2088

15. Kapandji M (1967) Treatment of urogenital prolapse by colpoisthmo-cystopexy with transverse strip and crossed, multiple layer, ligamento-peritoneal douglasorrhaphy. Ann Chir 21: 321-328

16. Bump RC, Mattiasson A, Bo K, Brubaker LP, DeLancey JO, Klarskov P et al (1996) The standardization of terminology of female pelvic organ prolapse and pelvic floor dysfunction. Am J Obstet Gynecol 175(1):10-17

17. CNGOF (Collège National des Gynécologues et Obstétriciens Français). (2011) Prevention of the complications related to the use of prosthetic meshes in prolapse surgery: guidelines for clinical practice-text of the guidelines. J Gynecol Obstet Biol Reprod (Paris) 40(8):851-853

18. Maher CF, Feiner B, DeCuyper EM, Nichlos CJ, Hickey KV, O'Rourke P (2011) Laparoscopic sacral colpopexy versus total vaginal mesh for vaginal vault prolapse: a randomized trial. Am J Obstet Gynecol. 204(4):360.e1-360.e7

19. Granese R, Candiani M, Perino A, Romano F, Cucinella G (2009) Laparoscopic sacrocolpopexy in the treatment of vaginal vault prolapse: 8 years experience. Eur J Obstet Gynecol Reprod Biol 146(2):227-231 
20. Higgs PJ, Chua HL, Smith AR (2005) Long term review of laparoscopic sacrocolpopexy. BJOG 112(8):1134-1138

21. Diwadkar GB, Barber MD, Feiner B, Maher C, Jelovsek JE (2009) Complication and reoperation rates after apical vaginal prolapse surgical repair: a systematic review. Obstet Gynecol 113(2 Pt 1):367-373

22. FDA safety communication: update on serious complications associated with transvaginal placement of surgical mesh for pelvic organ prolapse. Date issued: July13, 2011 http://www.fda. gov/MedicalDevices/Safety/AlertsandNotices/ucm262435.htm

23. Tan-Kim J, Menefee SA, Luber KM, Nager CW, Lukacz ES (2011) Prevalence and risk factors for mesh erosion after laparoscopic-assisted sacrocolpopexy. Int Urogynecol J 22(2):205-212 